\title{
Quelques aspects de la reproduction chez la chèvre rousse de Maradi. Comparaison avec d'autres races tropicales ou subtropicales
}

\author{
par J. B. HAUMESSER (*) \\ [avec la collaboration technique d'IBRAHIM ASSOUMANE (*) et de BARKE BOYI (*)]
}

\section{RESUME}

Le troupeau de chèvres rousses d'un village de la région de Maradi a été observé pendant trois années consécutives. Les résultats enregistrés ont permis de préciser quelques aspects de la reproduction chez cette race: répartition des mise-bas, âge à la première mise-bas, prolificité et intervalle entre les mise-bas, qui sont comparés à ceux d'autres races caprines tropicales ou subtropicales.

\section{INTRODUCTION}

Parmi les races de chèvres élevées dans la zone sahelo-soudanienne, la chèvre rousse de Maradi (Red Sokoto) mérite une attention particulière étant donné la valeur de sa peau; elle est réputée aussi pour sa prolificité et sa précocité. Une enquête a été entreprise pour préciser certains aspects encore assez peu connus de sa reproduction.

Les résultats obtenus sont comparés à ceux recueillis par divers auteurs pour d'autres races caprines vivant dans des zones tropicales ou subtropicales dont la plupart sont rassemblés par DEVENDRA et BURNS dans leur livre "Production of goat in the tropics» (6) mais, où malheureusement, ne figurent que très peu de données sur les races caprines du Sahel.

(*) I.E.M.V.T., Laboratoire de l'Elevage, B.P. 485, Niamey, République du Niger.

\section{MATERIEL ET METHODES}

L'enquête s'est déroulée du 15 septembre 1971 au 15 septembre 1974 dans un village de sédentaires hausas (Aderawa) situé à proximité de Maradi, sur un effectif moyen de 850 animaux. Un agent du Laboratoire, détaché en permanence dans ce village avait pour mission d'enregistrer les données concernant l'évolution du troupeau (mise-bas - mortalités abattages - ventes, etc.). Tous les animaux étaient marqués à l'oreille au moyen de marques numérotées en plastique. Au cours de la première année, un autre agent du Laboratoire, chargé de suivre l'évolution des troupeaux de deux autres villages proches l'un de l'autre, obtint des résultats semblables à ceux d'Aderawa. L'ensemble des données recueillies reflète donc ce qui se passe "en brousse " pour des animaux entretenus de façon traditionnelle.

La chèvre rousse de Maradi, son aire d'élevage et son exploitation ont déjà été largement décrites par DOUTRESSOULLE (8), ROTH 
(18), ROBINET (17), BEMBELLO (1) et MAINET (14). Seuls quelques points nouveaux seront donc précisés ci-après :

1. Le village où s'est déroulée l'enquête, situé sur l'isoyète 650 a vu sa pluviométrie singulièrement diminuer depuis 1967. (71: 398 $\mathrm{mm}, 72: 288 \mathrm{~mm}, 73: 350 \mathrm{~mm}, 74: 450 \mathrm{~mm}$.)

2. Pendant la période des cultures, les animaux du village sont gardés à l'attache dans les concessions afin d'éviter des dégâts. Au retour des champs, les propriétaires, dans la mesure du possible, leur amènent du fourrage (herbes de sarclage principalement). Les chèvres ne reçoivent en plus que le son de mil familial. C'est donc la plus mauvaise période pour les animaux du point de vue nutritionnel, ce qui se traduit par une augmentation des taux d'avortement et de mortalité néonatale (8, $12,13)$.

3. Tout le reste de l'année, les animaux errent en liberté autour du village dans un rayon de $3 \mathrm{~km}$, se nourrissant sur les chaumes et sur quelques arbustes. La ration des femelles pleines ou suitées est complétée quelques temps avec des fanes d'arachide et de wake (Vigna unguiculata).

4. La monte étant libre, effectuée par les jeunes mâles, le calcul des durées de gestation n'a pu être effectué.

Les différents calculs statistiques ont été effectués par le Service de Zootechnie du siège Central de l'I.E.M.V.T. à Paris, les intervalles de confiance des moyennes sont donnés à 95 p. 100.

\section{LA REPARTITION DES MISE-BAS AU COURS DE L'ANNEE}

La chèvre rousse de Maradi met bas tout au long de l'année (tabl. I) comme de nombreuses races tropicales ou subtropicales, au contraire des chèvres européennes qui ont une activité sexuelle toujours saisonnière (5). On sait que, le plus souvent, la durée de la saison sexuelle est d'autant plus longue que l'on se rapproche de l'équateur. "Son début se situe d'autant plus près du jour le plus long que l'amplitude de la photopériode est plus grande " (4).

Mais la durée de l'activité sexuelle dépend aussi de la race puisque, d'une part la chèvre alpine née et élevée en Guadeloupe conserve son activité sexuelle saisonnière de septembre à février (4) et que d'autre part, des chèvres de race naine africaine, importées depuis plusieurs générations en Allemagne et en Ecosse continuent à mettre bas tout au long de l'année (6).

Le tableau I montre qu'il existe deux maximums dans la fréquence des mise-bas comme l'avait déjà signalé BEMBELLO. A l'époque qui rassemble le maximum de mise-bas (plus de 50 p. 100 des mise-bas entre le 15 février et le 15 avril), correspondent des œstrus de mi-septembre à mi-novembre. Ceci peut être dû à un facteur climatique (fin des pluies) ou au lâcher de chèvres qui, au sortir d'une période de disette, vont pâturer sur les chaumes, trouvant alors une alimentation suffisante (flushing?).

Une fréquence analogue des mise-bas se retrouve chez plusieurs races tropicales ou subtropicales. Il en va ainsi pour la chèvre Beetal aux Indes (15) et pour la chèvre naine du Ghana (AMBLE et collab. 1964, cités par DEVENDRA). Par contre, pour la chèvre Jamnapari de l'Inde du nord (19) et pour la chèvre naine créole en Guadeloupe (3), les œstrus les plus fréquents se situent au début de la saison des pluies. D'après ROY et collab. (19) cette reprise d'activité sexuelle serait liée à la présence de substances œstrogéniques dans l'herbe jeune. Pour COGNIE et CORTEEL, s'y ajouterait l'effet du photopériodisme maximal à cette époque.

Ces deux facteurs pourraient être reponsables des ostrus de juillet avec le second sommet de mise bas beaucoup moins accusé mais significatif du 15 octobre au 15 novembre chez Ia chèvre rousse (entre $15 / 915 / 10$ et $15 / 10$ $15 / 11: t=4,07$; entre $15 / 1115 / 12$ et $15 / 10$ $15 / 11: t=5,03$ ). Ils pourraient influer aussi sur la venue en œstrus de la chèvre sahélienne qui, d'après Doutressoulle (cité par Devendra), ne mettrait bas qu'une fois par an, principalement en novembre.

\section{SEX RATIO

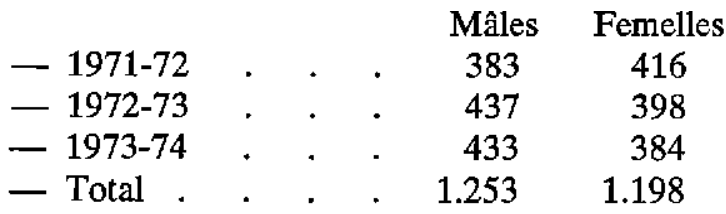

La proportion de mâles à la naissance (51 p. 100) est légèrement supérieure à celle 


\begin{tabular}{|c|c|c|c|c|c|c|c|c|c|c|c|c|c|c|c|c|}
\hline$z$ & $\angle b$ & $\varepsilon 89$ & $9 £ 6$ & $\varepsilon 0^{6} 0 \mp \angle \varpi^{6} \mathrm{~L}$ & $\lfloor\zeta \pitchfork Z$ & & & & & OOT & $899 \mathrm{~T}$ & & & & & \\
\hline 0 & 7 & $7 \varepsilon$ & $6 z$ & $S I^{*} 0 \mp \varepsilon 9^{*} \mathrm{~L}$ & $60 \mathrm{I}$ & $\varsigma z$ & $\angle T$ & $\angle 9$ & & $96^{\circ} 0 \mp 7$ & $\angle 9$ & $9 I$ & ot & $t h$ & & $6 / 5 I-8 / 5 \tau$ \\
\hline 0 & $\varepsilon$ & $\angle E$ & $s z$ & $7 L^{\circ} 0 \mp 99^{\circ} \mathrm{L}$ & $80 T$ & $\varsigma$ & $\angle 9$ & $9 \varepsilon$ & & $56^{\circ} 0 \mp 6^{\circ} \mathrm{E}$ & 59 & $\varepsilon$ & or & $z z$ & & $8 / \varsigma \mathrm{I}-L / \varsigma \mathrm{T}$ \\
\hline 0 & $\varepsilon$ & $o \varepsilon$ & $\angle S$ & $Z I^{\circ} 0 \mp 07^{*} \mathrm{~L}$ & $9 Z \Upsilon$ & 88 & 6 & $6 z$ & & II'I $\bar{\mp} \eta^{\prime} \varsigma$ & 06 & 79 & 8 & $\mathrm{BL}$ & & $L / S I-9 / 5 \mathrm{I}$ \\
\hline I & $\varsigma$ & 62 & $\angle E$ & $9 I^{6} 0 \mp 8 S^{6} \mathrm{~L}$ & $\gg \tau I$ & $z \varepsilon$ & $Z \mathrm{~L}$ & $0 L$ & & $66^{\circ} 0 \mp \varepsilon^{\circ} \eta$ & $Z L$ & $z z$ & 8 & $z \eta$ & & $9 / S I-S / S \tau$ \\
\hline$\tau$ & $z$ & 09 & 99 & $O \tau^{\circ} 0 \mp z S^{\circ} \mathrm{L}$ & $96 \mathrm{I}$ & $\tau \tau$ & 68 & 98 & & $O \varepsilon^{\prime} I \mp L L$ & $62 \mathrm{I}$ & $E I$ & 19 & s & & $\varsigma / \varsigma I-\eta / S I$ \\
\hline 0 & $L$ & $\varepsilon \mapsto \tau$ & IIE & $S 0^{\circ} 0 \mp 7 E^{\circ} \mathrm{L}$ & $8 \mathrm{~T} 9$ & $\eta \angle Z$ & $6 \subseteq \tau$ & $58 \mathrm{I}$ & & $8 I^{\circ} z+9^{\circ} \angle z$ & โ97 & $60 z$ & $60 \mathrm{I}$ & $\varepsilon \mapsto \tau$ & & $\eta / \varsigma I-\varepsilon / \varsigma \tau$ \\
\hline 0 & $\eta T$ & $6 S \mathrm{~L}$ & $6 z z$ & $90^{\circ} 0 \mp \angle 7^{\circ} \mathrm{L}$ & 685 & $\varsigma \succsim 乙$ & $z 9 z$ & $20 \mathrm{~T}$ & & $60^{\circ} z \mp I^{6} \eta Z$ & 207 & โ9โ & $\eta \angle T$ & $\angle 9$ & & $E / \varsigma \mathrm{I}-\tau / \varsigma \mathrm{L}$ \\
\hline 0 & 0 & $6 \mathrm{I}$ & 27 & ZI'o $\mp I E^{\star} \mathrm{I}$ & 08 & 7 & $Z L$ & $\eta$ & & $16^{\circ} \circ \mp 9^{\prime \prime} \varepsilon$ & โ9 & $\tau$ & SS & 7 & & $\tau / \varsigma I-\tau / \varsigma \mathrm{I}$ \\
\hline 0 & $\mathrm{I}$ & $\angle \mathbf{I}$ & St & $0 z^{\circ} 0 \mp 8 S^{\circ} \tau$ & $z s$ & II & I $\varepsilon$ & ot & & $\angle 9^{\circ} 0 \mp 6^{\circ} \mathrm{I}$ & $\varepsilon \varepsilon$ & 9 & $I z$ & 9 & & $\tau / \zeta \tau-z T / S T$ \\
\hline 0 & I & It & $\angle Z$ & $2 I^{\circ} 0 \mp 29^{\circ} \mathrm{I}$ & $z I I$ & & 88 & 9 & $8 \mathrm{~T}$ & $\angle 6^{\circ} 0 \mp I^{\circ} \eta$ & 69 & & $\varepsilon S$ & 7 & $2 \mathrm{I}$ & $\tau I / S I-I I / S I$ \\
\hline 0 & 7 & 59 & $0 L$ & $60^{\circ} 0 \rightleftarrows \varepsilon 5^{\circ} \mathrm{L}$ & $2 I z$ & & $\tau \varepsilon$ & $\angle L$ & $\varepsilon O I$ & $\varsigma \varepsilon^{\circ} I \mp \varepsilon^{\prime} 8$ & $6 E I$ & & 22 & 95 & โ9 & $I I / S I-O I / S T$ \\
\hline 0 & $\varepsilon$ & 67 & 82 & $2 I^{\circ} \mathrm{O} \mp 69^{\circ} \mathrm{L}$ & SEI & & $\tau \tau$ & $\pitchfork \varepsilon$ & 68 & $50^{\circ} \mathrm{T} \mp 8^{\circ} \mapsto$ & 08 & & $L$ & $6 \mathrm{I}$ & 75 & $0 T / \varsigma \tau-6 / \subseteq T$ \\
\hline 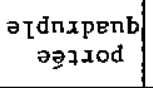 & 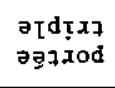 & $\begin{array}{l}\text { zIqnop } \\
\text { zəżIod }\end{array}$ & 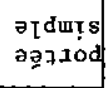 & \multirow{2}{*}{ 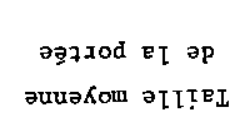 } & $\mathrm{I}$ & $7 \angle 6 \tau$ & $\varepsilon \angle 6 \mathrm{I}$ & $Z \angle 6 \mathrm{I}$ & $\mathrm{T} \angle 6 \mathrm{~T}$ & \multirow{2}{*}{ 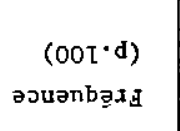 } & I & $7 \angle 6 I$ & $\varepsilon \angle 6 \mathrm{I}$ & $\tau \angle 6 \mathrm{I}$ & $\mathrm{I} \angle 6 \mathrm{~T}$ & \multirow[t]{2}{*}{ әрот̣дอd } \\
\hline \multicolumn{4}{|c|}{ 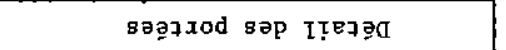 } & & \multicolumn{5}{|c|}{ 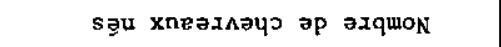 } & & \multicolumn{5}{|c|}{ seq-asṭడ ap aIqmoN } & \\
\hline
\end{tabular}


des femelles comme chez la plupart des mammifères; on ne rencontre pas d'intersexués; les deux sexes chez la chèvre rousse sont cornus.

\section{Age à la première mise-bas}

Calculé sur 227 intervalles entre la naissance et la première mise-bas, l'âge moyen à la première mise-bas pour la chèvre rousse se situe vers 14 mois $(426,7 \pm 13,5 \mathrm{j} ; \mathrm{V}=23,8 \mathrm{p}$. 100 ), ce qui la met en bonne position par rapport à la plupart des races tropicales ou subtropicales, et confirme sa précocité sexuelle.

COGNIE et collab. (2) situent l'âge de la première mise-bas entre 11 et 15 mois pour la chèvre créole en Guadeloupe. EPSTEIN et HERZ en Israël (9) donnent pour les races Syrienne des montagnes, Damas et Maltaise respectivement l'âge de 12 mois, 24 mois, 12 mois.

DEVENDRA rapporte des âges plus élevés : 19 mois pour la race locale d'Ouganda et celle de Ceylan (SACHER et TRAIL), 22 mois pour la Beetal (KAURA), tout en précisant qu'il ignore s'il s'agit de monte naturelle ou de période de saillie volontairement retardée pour que l'animal ait atteint un poids suffisant.

A ce sujet il faut noter qu'en Europe à l'heure actuelle, on considère qu'une chevrette doit peser 60 p. 100 de son poids adulte au moment de la saillie.
Les propriétaires hausas, qui sont presque exclusivement des femmes, semblent ignorer, apparemment sans dommage, ce principe qui, d'ailleurs, n'est peut-être pas applicable à la chèvre rousse. En effet, elles commercialisent en priorité après les mâles la plupart des chevrettes n'ayant pas mis bas à l'âge de 18 mois, ce qui constitue une sélection sur la précocité sexuelle.

\section{PROLIFICITE}

Le tableau I présente les résultats obtenus au cours des trois années d'enquête.

Définie par la taille moyenne de la portée, la prolificité de la chèvre rousse de Maradi $(1,47 \pm 0,03)$ semble moyenne comparée aux données du tableau II emprunté à DEVENDRA auquel sont ajoutés les résultats de COGNIE (2). Mais il faut remarquer qu'excepté les données relatives aux chèvres créole et syrienne des montagnes récoltées sur le terrain, il s'agit de résultats obtenus en station où l'alimentation peut être supposée rationnelle.

La taille moyenne de la portée varie chez la chèvre, au sein d'une même race, en fonction de l'âge, du poids, et du rang de mise-bas.

$\mathrm{Ne}$ disposant pas d'assez d'animaux d'âge connu, seul le rang de mise-bas a pu être étudié.

TABL. $N^{\circ}$ II-Prolificité de quelques races de chèvres (taille de la portëe) (DEVENDRA et BURNS)

\begin{tabular}{|c|c|c|c|}
\hline $\mathrm{R} a \mathrm{ce}$ & $\mathrm{L} i \mathrm{e} u$ & $\begin{array}{c}\text { Taille moyenne } \\
\text { de la portée }\end{array}$ & $\mathrm{R}$ é $\mathrm{f} \ddot{\mathrm{e}} \mathrm{I} \mathrm{e} \mathrm{c} \mathrm{e}$ \\
\hline $\begin{array}{l}\text { Race crëole de la Guadeloupe } \\
\text { Maltaise et Damas } \\
\text { Damas } \\
\text { Kilis } \\
\text { Kambing Katjang } \\
\text { Anglo nubienne } \\
\text { Crolsements divers } \\
\text { Ma t' ou } \\
\text { Syrienne des montagnes } \\
\text { Negev } \\
\text { Appenzeller } \\
\text { Saanen } \\
\text { Damas } \\
\text { Anglo nubienne } \\
\text { Maltaise } \\
\text { Anglo nubienne } \\
\text { Anglo nubienne x race locale } \\
\text { Noire du Bengale }\end{array}$ & $\begin{array}{l}\text { Guadeloupe } \\
\text { Athalasaa (Chypre) } \\
\text { Chypre } \\
\text { Ankara (Turquie) } \\
\text { ) Serlang (Malaisie) } \\
\text { Province de Hupey } \\
\text { (Chine) } \\
\text { ) } \\
\text { ) Rehovot (Isrä1) } \\
\text { ) } \\
\text { )Palmar (Ile Maurice) } \\
\text { Haringhata (Inde) }\end{array}$ & $\begin{array}{c}1,91 \\
1,85 \\
1,71 \\
1,27 \\
1,65 \\
1,43 \\
1,67 \text { a, } 2,06 \\
2,24 \\
1,44 \\
1,38 \\
1,27 \\
1,90 \\
1,76 \\
1,75 \\
1,59 \\
2,29 \\
2,45 \\
2,04\end{array}$ & 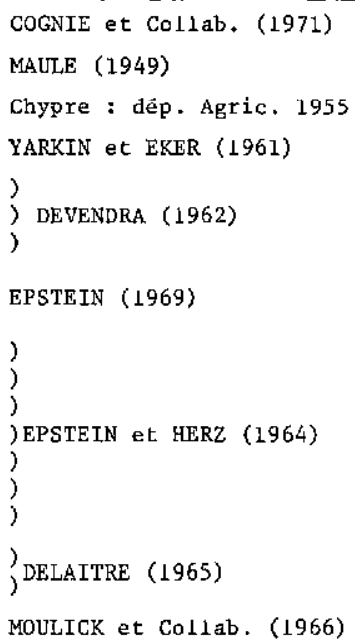 \\
\hline
\end{tabular}


TABl. N`III-Augmentatıon de la taille moyenne de la portêe avec le rang de mise-bas.

\begin{tabular}{|c|c|c|c|c|c|}
\hline & \multicolumn{2}{|c|}{ Chèvre crëole de la Guadeloupe } & \multicolumn{3}{|c|}{ Chèvre rousse de Maradi } \\
\hline & $\begin{array}{l}\text { Taille moyenne } \\
\text { de } 12 \text { portée }\end{array}$ & $\begin{array}{c}\text { Nombre } \\
\text { d'observations }\end{array}$ & $\begin{array}{l}\text { Taille moyenne } \\
\text { de la portée }\end{array}$ & $\begin{array}{c}\text { Nombre } \\
\text { d'observations }\end{array}$ & $\mathrm{r}$ \\
\hline $\begin{array}{l}\text { Première } \\
\text { mise-bas }\end{array}$ & 1,67 & $?$ & $1,08 \pm 0,04$ & 227 & \multirow{3}{*}{$\begin{array}{l}2,033 \\
19,753 \\
8,347\end{array}$} \\
\hline $\begin{array}{l}\text { Deuxième } \\
\text { mise-bas }\end{array}$ & 2,12 & 34 & $1,20+0,11$ & 51 & \\
\hline $\begin{array}{l}\text { Troisième } \\
\text { mise-bas et } \\
\text { plus }\end{array}$ & 2,33 & 30 & $1,72+0,05$ & 483 & \\
\hline
\end{tabular}

La taille moyenne de la portée augmente bien avec le rang de mise-bas comme le montre le tableau III sur lequel sont inscrits aussi les résultats de Cognie. Il existe une différence significative entre les taux de prolificité observés.

\section{INTERVALLES ENTRE LES MISE-BAS}

La durée de l'intervalle entre deux mise-bas constitue un critère intéressant de la fertilité des femelles. La durée de gestation étant relativement constante et connue (5 mois) c'est l'intervalle compris entre la mise-bas et la nouvelle fécondation (ou "période de service ") qui est la plus susceptible de variations.

\section{Moyenne générale}

Calculée sur 665 intervalles, la moyenne est de $332,4 \pm 6,2 \mathrm{j}$ (environ 11 mois). En éliminant les intervalles entrecoupés d'avortements sur 633 observations, la moyenne est de 326,4 $\pm 5,7 \mathrm{j}$. Il n'y a pas de différence significative avec la moyenne générale $(t=1,43)$.

Le graphique montre l'histogramme des valeurs des intervalles entre les mise-bas.

Moins de 12 p. 100 des intervalles sont inférieurs à 8 mois, ce qui peut-être considéré comme bon pour des races de chèvres à activité sexuelle continue.

43,5 p. 100 sont compris entre 240 et 340 jours, ils sont considérés comme moyens; 45,2 p. 100 sont considérés comme mauvais car dépassant 340 jours.

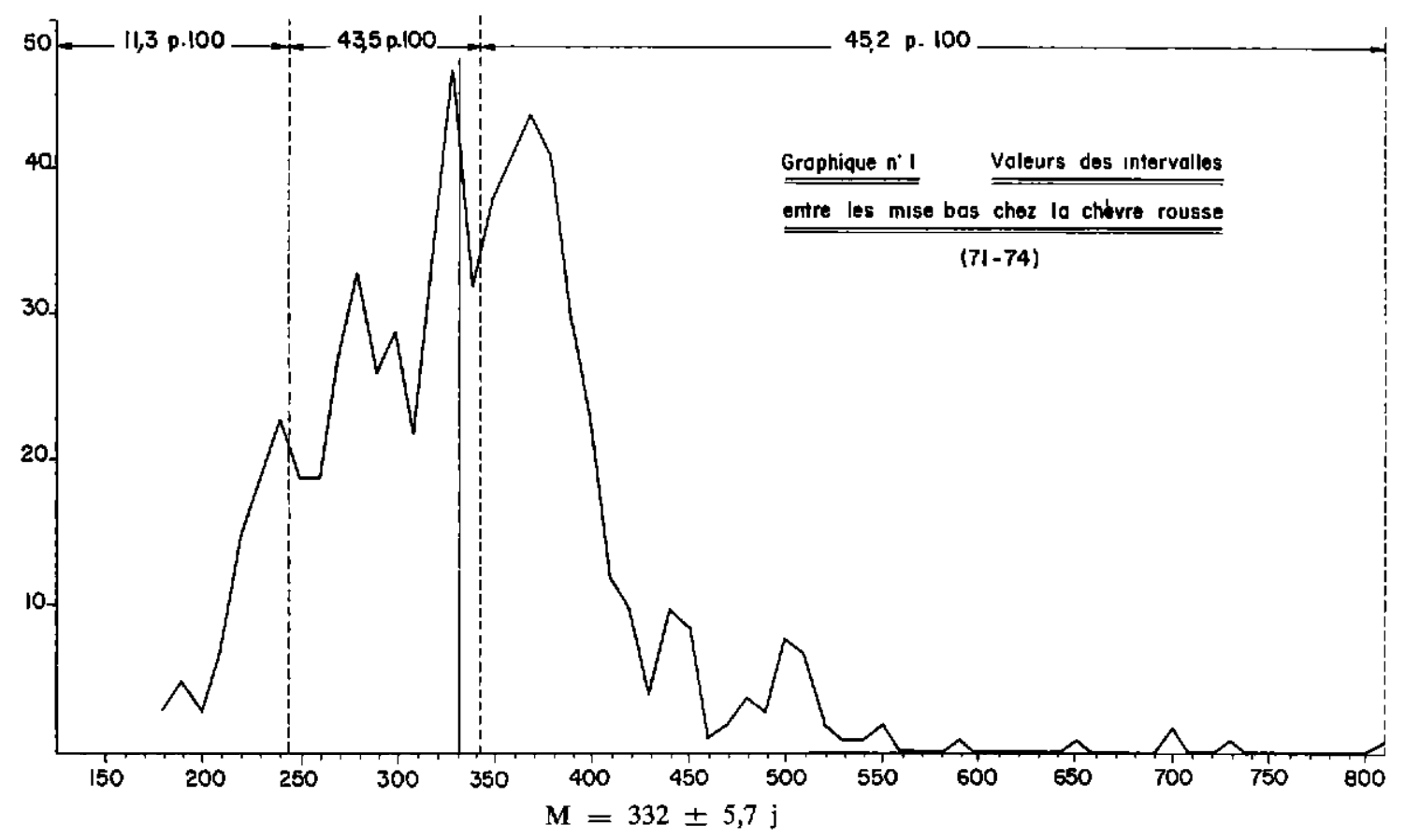


TABL. $N^{\circ}$ IV - "Période de service" durée de la gestation et intervalles entre les mise-bas pour quelques races et leurs croisements (DEVENDRA et BURNS)

\begin{tabular}{|c|c|c|c|c|c|}
\hline$R$ a $\subset \mathrm{e}$ & $\mathrm{R} \overrightarrow{\mathrm{e}} \mathrm{g}$ i $\circ \mathrm{n}$ & $\begin{array}{l}\text { Période } \\
\text { de } \\
\text { service } \\
\text { (jours) }\end{array}$ & $\begin{array}{l}\text { Durée } \\
\text { de 1a } \\
\text { gestation } \\
\text { (jours) }\end{array}$ & $\begin{array}{l}\text { Intervalle } \\
\text { entre les } \\
\text { mise-bas } \\
\text { (jours) }\end{array}$ & $A u t e u r s$ \\
\hline Jamnapari & \}nited Provinces & - & 150 & - & JSLATER et BHATIA \\
\hline Barbarie & $\{$ (Inde) & - & 146 & - & )$(1935)$ \\
\hline Barbarie & ) & - & 146 & - & ) \\
\hline Jamnapari & ) Etah. (Inde) & - & 150 & - & JKAURA (1943) \\
\hline Beetal & 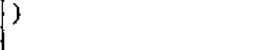 & - & 147 & - & ) \\
\hline Race locale & Hissar (Inde) & $90-120$ & - & $240-270$ & KARTHA (1937) \\
\hline Saanen & ) & - & 152 & - & ) \\
\hline Toggenburg & )Agua Branca & - & 151 & - & ) SANTIAGO (1946) \\
\hline $\begin{array}{l}\text { Anglo nubienne } \\
\text { Race locale }\end{array}$ & )$^{(\text {Brësil) }}$ & $\overline{-}$ & $\begin{array}{l}148 \\
145\end{array}$ & - & ) \\
\hline Phillpine & $\begin{array}{l}\text { Laguna } \\
\text { (Philliprnes) }\end{array}$ & - & 148 & - & GALEON (1951) \\
\hline Fidji & Sigatoka (Fidji) & - & - & 262 & $\begin{array}{l}\text { PAYNE et MILES } \\
\text { (1953) }\end{array}$ \\
\hline Race Locale & Bas Congo & - & $145-148$ & - & HENROTTE (1961) \\
\hline Race Locale & ) & 92 & 148 & 240 & ) \\
\hline Anglo nublenne $\times 1 / 2$ locale & Derdang & 203 & 148 & 351 & \\
\hline $\begin{array}{l}\text { Ang10 nubienne } \times 1 / 4 \text { locale } \\
\text { Anglo nubienne }\end{array}$ & )(MaLaisie) & $\begin{array}{l}204 \\
327\end{array}$ & 147 & $\begin{array}{l}357 \\
480\end{array}$ & ) DEVENDRA (1962) \\
\hline Anglo nubienne & 1 & & 153 & 480 & ) \\
\hline $\begin{array}{l}\text { Anglo nubienne } \\
\frac{7}{8} \text { Anglo nubienne } \times \frac{1}{8} \text { locale }\end{array}$ & $\begin{array}{l}\text { Palmar (Ile } \\
\text { Maurice) }\end{array}$ & - & $\overrightarrow{-}$ & $\begin{array}{l}363 \\
372\end{array}$ & $\{$ DELAITRE (1965) \\
\hline Race locale & Ghana & - & $145-153$ & - & OPPONG (1965) \\
\hline Afrique de L'Est:Race Locale & Serere (Ouganda) & - & 147 & & WLLSON (1957) \\
\hline Mubende & Mbarara (Ougande) & - & - & 297 & $\begin{array}{l}\text { SAKER et TRAIL } \\
(1966)\end{array}$ \\
\hline Inde du Sud: Race locale & $\begin{array}{l}\text { Kottukachehiya } \\
\text { (Ceylan) }\end{array}$ & - & - & $32 B$ & WIJERATNE (196B) \\
\hline Noire du Bengale & Haringhata (Inde) & - & 144 & - & $\begin{array}{l}\text { GUPTA et Collab. } \\
(1964)\end{array}$ \\
\hline Crëole de la Guadeloupe & Guadeloupe & - & - & 240 & $\begin{array}{l}\text { COGNIE et Collab. } \\
\text { (1971) }\end{array}$ \\
\hline
\end{tabular}

Le tableau IV emprunté à DEVENDRA donne quelques valeurs d'intervalles pour des races caprines et leur croisement. Toutes les races locales des zones tropicales ont des intervalles inférieurs à celui observé chez la chèvre rousse de Maradi. Mais il s'agit de races élevées en zone humide, ce qui doit diminuer grandement l'influence du niveau nutritionnel sur la durée de la " période de service ».

Chez les races locales croisées avec l'anglonubienne, sexuellement saisonnée, on constate un allongement de l'intervalle entre les misebas.

\section{Darée des intervalles en fonction du mois de naissance des chevreaux}

Le tableau VI donne les durées des intervalles en fonction du mois de naissance des chevreaux (calculées avec les avortements).

Le tableau $\mathrm{V}$ donne les valeurs de $\mathrm{t}$ calculées en comparant les moyennes observées deux à deux.

Au point de vue durée des intervalles, on peut distinguer trois périodes:

$-1^{\text {re }}$ période: septembre à avril.

Les mise-bas survenant pendant cette période sont suivies d'un intervalle entre les 
TABL. $\mathrm{N}^{0} \mathrm{~V}$-Comparaison des moyennes observées deux à deux.

\begin{tabular}{|c|c|c|c|c|c|c|c|c|c|c|c|c|}
\hline & 1 & 2 & 3 & 4 & 5 & 6 & 7 & 8 & 9 & 10 & 11 & 12 \\
\hline 1 & & $-0,30$ & 0,11 & 0,087 & 2,86 & 1,77 & 4,74 & 5,09 & 1,33 & 0,87 & 0,87 & 1,22 \\
\hline 2 & & & 0,88 & 0,72 & 6,18 & 2,63 & 7,97 & 9,21 & 1,89 & 1,46 & 1,42 & 1,49 \\
\hline 3 & & & & 0,032 & 6,75 & 2,33 & 8,24 & 9,87 & 1,60 & 1,11 & 1,08 & 1,29 \\
\hline 4 & & & & & 5,51 & 2,25 & 7,48 & 8,68 & 1,57 & 1,07 & 1,05 & 1,28 \\
\hline 5 & & & & & & $-0,52$ & 3,61 & 4,39 & $-0,78$ & $-1,78$ & $-1,67$ & $-0,17$ \\
\hline 6 & & & & & & & 2,65 & 2,92 & $-0,28$ & $-0,93$ & $-0,88$ & 0,093 \\
\hline 7 & & & & & & & & 0,21 & $-2,61$ & $-3,82$ & $-3,64$ & $-1,38$ \\
\hline 8 & & & & & & & & & $-2,83$ & $-4,16$ & $-3,94$ & $-1,47$ \\
\hline 9 & & & & & & & & & & $-0,56$ & $-0,53$ & $-0,27$ \\
\hline 10 & & & & & & & & & & & 0,020 & 0,67 \\
\hline 11 & & & & & & & & & & & & 0,65 \\
\hline 12 & & & & & & & & & & & & \\
\hline
\end{tabular}

TABL.N*VI-Moyenne en fonction du mois de mise-bas

\begin{tabular}{|l|c|c|c|}
\hline $\begin{array}{c}\text { Mois de } \\
\text { mise-bas }\end{array}$ & Moyenne & $\begin{array}{c}\text { Nombre } \\
\text { observations }\end{array}$ & \\
\hline Septembre & $314,7 \pm 39,0$ & 35 \\
Octobre & $328,7 \pm 31,5$ & 37 \\
Novembre & $328,2 \pm 33,4$ & 39 \\
Décembre & $304,3 \pm 83,8$ & 6 \\
Janvier & $348,6 \pm 34,2$ & 22 \\
Février & $353,9 \pm 13,7$ & 101 \\
Mars & $346,8 \pm 8,5$ & 151 \\
Avril & $347,0 \pm 13,3$ & 161 \\
Mai & $298,9 \pm 11,3$ & 35 \\
Juin & $307,7 \pm 33,6$ & 22 & 2e période \\
Juillet & $257,4 \pm 20,4$ & 28 & 1ère période \\
Août & $254,7 \pm 17,0$ & 28 & 3e période \\
\hline
\end{tabular}

mise-bas de :

$$
343,2 \pm 6,6 \text { jours }(\mathrm{N}=552)
$$

- $2^{\mathrm{e}}$ période : mai, juin :

$$
302,3 \pm 14,3 \text { jours }(\mathrm{N}=57)
$$

- $3^{\mathrm{e}}$ période : juillet, août :

$$
266,1 \pm 13,0 \text { jours }(\mathbf{N}=56)
$$

La durée des intervalles diminue à mesure que la période de mise-bas se rapproche de la période d'activité sexuelle la plus intense (miseptembre à mi-novembre).

\section{Durée des intervalles pour les femelles dont les portées sont mortes dans les 15 premiers jours de la vie}

Calculée sur 32 intervalles, la moyenne est de $268,8 \pm 22,1$ j.
Il y a une différence significative avec la moyenne générale $(\mathrm{t}=5,23)$, la moyenne de la $1^{\text {re }}$ période $(t=6,47)$, et la moyenne de la $2^{\mathrm{e}}$ période $(t=2,54)$. Il n'y en a pas avec la moyenne de la $3^{\mathrm{e}}$ période $(\mathrm{t}=0,99)$.

L'allaitement des chevreaux allongerait la " période de service ", mais il faut remarquer que la majorité des cas de mortalité néonatale survient pendant la saison des cultures qui correspond aussi à la troisième période, où les intervalles sont plus courts.

\section{Intervalles entre les avortements et les mise-bas suivantes}

A la suite de 59 avortements, les mise-bas ont eu lieu $219,8 \pm 15,6$ jours plus tard. Il y a une différence significative avec les intervalles entre les mise-bas $3^{e}$ période $(t=3,52)$ et par suite avec toute les autres moyennes observées.

Il a déjà été démontré $(10-13)$ que la plupart des avortements chez la chèvre rousse de Maradi reconnaissaient une étiologie nutritionnelle. Ceci est confirmé par la brièveté de la "période de service " leur succédant. L'involution utérine, la fécondation et la nidation ne sont pas retardées par un processus infectieux au niveau du tractus génital (21).

Il faut noter que d'une part les chèvres ayant avorté ne sont jamais traites, ce qui peut favoriser le raccourcissement de la "période de service " et que, d'autre part, comme dans les 
cas de mortalité néonatale, les avortements surviennent surtout au cours de la $3^{\mathrm{e}}$ période où la moyenne des intervalles est la plus courte.

\section{APPRECIATION DE LA VALEUR REPRODUCTIVE \\ DE LA CHEVRE ROUSSE}

La production annuelle d'un troupeau de chèvres reproductrices cumule les résultats :

- du taux de fertilité annuelle;

- du taux de fécondité annuelle;

- du taux de prolificité (cf. chapitre prolificité);

- du taux de productivité annuelle;

les résultats obtenus sont inscrits au tableau VII.

\section{Taux de fécondité annuelle}

Il est défini par le rapport :

Nombre de chevreaux nés au cours de l'année

Nombre de reproductrices présentes dans le troupeau

Ce taux, qui est de 167 p. 100 pour les trois années considérées, ne varie pas significativement d'une année à l'autre $\left(\chi^{2}=2,11\right)$.

Le taux de fécondité annuelle est plus intéressant à connaître que les taux de fertilité et de prolificité car il traduit la production d'une femelle dont les intervalles sont réduits, aussi bien que celle d'une femelle prolifique.

\section{Taux de prolificité annuelle}

La prolificité de la chèvre rousse a déjà été

TABL. $N^{\circ}$ VII- Performances reproductives de la chèvre rousse de Maradi.

\begin{tabular}{|c|c|c|c|c|}
\hline & $1971 / 72$ & $1972 / 73$ & $1973 / 74$ & $\begin{array}{c}\text { Moyenne sur les } \\
3 \text { années }\end{array}$ \\
\hline $\begin{array}{l}\text { Taux de fertilité annuelle (p.100) } \\
\text { Nambre de mise-bas } \\
\text { Nombre de productrices }\end{array}$ & $\frac{525}{457}=114$ & $\frac{565 \times 100}{505}=112$ & $\frac{578 \times 100}{500}=115$ & $\frac{1668 \times 100}{1462}=114$ \\
\hline $\begin{array}{l}\text { Taux de fécondité annuelle (p.100) } \\
\frac{\text { Nombre de chevreaux nés }}{\text { Nombre de reproductrices }} \times 100\end{array}$ & $\frac{799}{457}=174$ & $\frac{835 \times 100}{505}=165$ & $\frac{817 x}{500}+100=163$ & $\frac{2451 \times 100}{1462}=167$ \\
\hline $\begin{array}{l}\text { Taux de prolificité annue1le (p.100) } \\
\frac{\text { Nombre de chevreaux nés }}{\text { Nombre de mise-bas }} \times 100\end{array}$ & $\frac{799}{525}=152$ & $\frac{835 \times 100}{565}=148$ & $\frac{817 \times 100}{57 B}=141$ & $\frac{2415 \times 100}{1668}=147$ \\
\hline $\begin{array}{l}\text { Taux de productivité annuelle (p.100) } \\
\text { Nombre de chevreaux vivants à } 6 \text { nois } \\
\text { Nombre de reproductriceg }\end{array}$ & $\frac{690}{457}=151$ & $\frac{708 \times 100}{505}=140$ & $\frac{659 \times 100}{500}=131$ & $\frac{2057 \times 100}{1462}=140$ \\
\hline
\end{tabular}

\section{Taux de fertilité annuelle}

Il est défini par le rapport :

Nombre de chèvres ayant mis-bas au cours de l'année

Nombre de reproductrices présentes dans le troupeau

Ce taux est de 114 p. 100 pour les 3 années considérées. Il ne varie pas significativement d'une année à l'autre $\left(\chi^{2}=0,34\right)$, et ne diffère par significativement de celui obtenu par l'intervalle moyen entre les mise-bas rapporté à l'année : $\frac{365 \times 100}{322.4}=109,8$ p. 100 . envisagée au chapitre prolificité. Le taux de prolificité annuelle (147 p. 100) pour les trois années ne varie pas significativement d'une année à l'autre $\left(\chi^{2}=1,36\right)$.

\section{Taux de productivité annuelle}

C'est le taux de sevrage, qui est défini par le rapport :

Nombre de chevreaux vivant à 6 mois

Nombre de reproductrices présentes $\times 100$ dans le troupeau

Le sevrage s'effectue spontanément vers l'âge de 5 à 6 mois, et la majorité des cas de mortalité survient avant l'âge de 6 mois. Ce taux 
est très intéressant à connaître chez les races de chèvres pour qui la production laitière est secondaire comme c'est le cas de la chèvre rousse de Maradi.

Le taux de productivité annuelle est de 140 p. 100 pour les trois années considérées.

Il n'y a pas de différence significative entre les années $71 / 72$ et $72 / 73\left(\chi^{2}=1,92\right)$ ni entre les années $72 / 73$ et $73 / 74\left(\chi^{2}=1,30\right)$. Mais il y en a une entre les années $71 / 72$ et $73 / 74$ $\left(\chi^{2}=6,23\right)$. Cette augmentation de la mortalité avant sevrage peut être due à l'augmentation de la pluviosité (72:288 mm; $74: 450 \mathrm{~mm}$ ) et par suite du parasitisme gastro-intestinal (13).

\section{CONCLUSION}

La chèvre rousse de Maradi, élevée de manière traditionnelle, présente des performances reproductives moyennes.

La première mise-bas survient à l'âge de 14 mois, l'intervalle entre les mise-bas est de 11 mois avec une prolificité de 1,5 chevreau par portée.

Il faut souligner que, durant les trois années d'enquête, la pluviosité était nettement inférieure à la moyenne.
Des enquêtes ont débuté chez les autres races caprines du Niger sédentaire et nomade afin de les comparer à la chèvre rousse au point de vue de la reproduction.

Il est probable qu'une alimentation suffisante et équilibrée améliorerait les performances de la chèvre rousse. L'intervalle entre les mise-bas pourrait être ramené à 8 mois ( 3 mise-bas en 2 ans) et la taille moyenne de la portée pourrait s'élever à deux chez les multipares.

L'extériorisation des potentialités génétiques de la chèvre rousse sur le plan des phénomènes de la reproduction, en lui apportant une alimentation rationnelle, est un des objectifs que s'est fixé le centre caprin de Maradi.

\section{Remerciements}

L'auteur remercie vivement :

- M. COUlOMB du Siège Central de 1'I.E.M.V.T. qui a effectué l'interprétation statistique des résultats.

- MM. Y TOUNKARA, O. MOUSSA et RABO ALLAO qui se sont succédés a à la Direction Départementale de l'Elevage à Maradi, pour leur contribution à l'enquête.

\title{
SUMMARY
}

Some aspects of reproduction in the reddish brown goats of Maradi. Comparison with other tropical or sub-tropical breeds

Observations have been made for three successive years on a flock of reddish brown goats in a village of the Maradi area. From the results obtained, it was possible to define more accurately some aspects of reproduction in this breed: distribution of kidding, age at the first kidding, reproduction rate, and intervals between kidding, aspects which are compared with those of other tropical or sub-tropical goat breeds.

\section{RESUMEN}

\begin{abstract}
Algunos aspectos de la reproducción en la cabra bermeja de Maradi. Comparación con otras razas tropicales o subtropicales
\end{abstract}

Se ha observado durante tres años seguidos el rebaño de cabras bermejas de una aldea en la región de Maradi (Niger). Los resultados permitieron precisar algunos aspectos de la reproducción en dicha raza: repartíción de los partos, edad al primer parto, fertilidad e intervalo entre los partos, que se comparan con los de otras razas cabrunas tropicales o subtropicales. 


\section{BIBLIOGRAPHIE}

1. BEMBELLO (H.). La chèvre rousse et son exploitation au Niger. Thèse Doct. Vét. Toulouse 1961, $\mathrm{n}^{\circ} 17$.

2. COGNIE (Y.), HOUIX (Y.), LOGEAY (B.). Données sur la croissance et la reproduction de la chèvre créole en Guadeloupe. In : $2^{\mathbf{e}}$ conférence internationale de l'élevage caprin. Tours 17 . 19 juillet 1971.

3. COGNIE (Y.), CORTEEL (J. M.). 1970. Résultats non publiés.

4. CORTEEL (J.M.). La maîtrise du cycle sexuel chez la chevrette et la chèvre. Nouzilly, I.N.R.A., 1971.

5. CORTEEL (J.M.). Quelques aspects physiologiques et techniques de la reproduction chez les caprins et les ovins. Milan, Institut Lazarro Spallanzani, Conférences 16 et 19 avril 1974.

6. DEVENDRA (C.), BURNS (M.). Goat production in the tropics. Farhnam Royal, C.A.B., 1970.

7. DOUTRESSOULLE (G.). L'élevage au Niger. Thèse Doct. Vét. Toulouse, 1974.

8. DOUTRESSOULLE (G.). L'élevage en A.O.F. Paris, Larose, 1947.

9. EPSTEIN (H.), HERZ (A.). Fertility and birth weights of goat in a subtropical environment. J. Agric. Sci., Camb., 1964, 62: 237-244. Anim. Breed Abstr., 1964, 32: 505 (Abstr. no 3, 116).

10. HAUMESSER (J. B.), POUTREL (B.). Contribution à l'étude des Rickettsioses au Niger. Enquête épidémiologique réalisée dans la région de $\mathrm{Ma}$ radi. Rev. Elev. Méd. vét. Pays trop., 1973, 26 (3): 293-298.

11. I.E.M.V.T. Laboratoire de l'Elevage de Niamey: rapport annuel 1971.

12. I.E.M.V.T. Laboratoire de l'Elevage de Niamey: rapport annuel 1972.
13. I.E.MV.T. Laboratoire de l'Elevage de Niamey : rapport annuel 1973.

14. MAINET (G.). L'élevage dans la région de Maradi. Cah. Outrem., 1965 (69).

15. MISHRA (M. R.), BISWAS (S. C.). A study on distribution of cestrus in Deshi goats. Ind. J. dairy Sci, 1966, 19 (3): 132-134. Anim. Breed Abstr., 1968, 36: 92 (Abstr. to 525).

16. MOULICK (S. K.), GUHA (H.), GUPTA (S.) et Collab. Factors affecting multiple birth in black bengal goats. Ind. J. vet. Sci., 1966, 36 (3) : 154163. Anim. Breed Abstr., 1967, 35: 275 (Abstr. $\mathrm{n}^{\mathrm{o}}$ 1560).

17. ROBINET (A. H.). La chèvre rousse de Maradi, son exploitation et sa place dans l'économie et l'élevage de la République du Niger. Rev. Elev. Méd. vét. Pays trop., 1967, 20 (1) : 129-186. Anim. Breed Abstr., 1967, 35: 635 (Abstr. n० 3746 ).

18. ROTH (M.). La petite chèvre rousse du Niger. Bull. Serv. zootech. épizoot. Afr. Occ. Fr., 1938, 1 (1) : 13-19.

19. ROY (A.) et Collab. Studies on certain aspects of sheep and goat husbandry : artificial breeding, telescoping the breeding season and certain reproductive phenomena in sheep and goats. Ind. $I$. vet. Sci., 1962, 32 (5) : 269-275.

20. SHELTON (M.). Factors affecting kid production of Angora does. Tex. Agric. Exp. Stn. Misc. Publ., 1961 (MP 496). Anim. Breed Abstr., 1962, 30 : 518 (Abstr. n० 2 641).

21. VAN HEERDEN (K. M.). Investigations into the cause of abortions in angora goat in South Africa. Onderstepoort J. vet. Res., 1963, 30 (1): $23-83$. Anim. Breed. Abstr., 1964, 32 : 51 (Abstr. 口$^{0}$ 321). 